\title{
Investigation of GOSIP Technology at ANL
}

By

Linda Winkler

January 1992

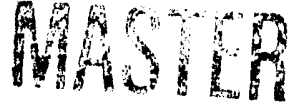

Computing and Telecommunications Division

Argonne National Laboratory, 9700 South Cass Avenue, Argonne, Illinois 60439-4801

(Intended primarily for internal distribution) 


\section{Acknowledgments}

The work presented in this document was made possible by funding supplied by the U.S. Department of Energy. We would like to thank Steve Hackman for his support and encouragement. We hope that this work provides valuable information and expertise to the DOE community in the area of Open Systems Interconnection. We plan to continue to investigate other aspects of GOSIP technology and make our findings widely available. 


\section{CONTENTS}

Executive Summary vii

1.0 introduction 1

2.0 The GOSIP Testing Environment at Argonne 3

3.0 Network Services 5

3.1 GOSIP Alternatives 5

3.2 OSI Addressing 6

3.3 Configuration 8

3.3.1 SunNet OSI 8

3.3.2 IBM OSIMF/6000 9

3.3.3 Cisco AGS+ 9

3.4 Management 9

3.4.1 SunNet OSI 9

3.4.2 IBM OSIMF/6000 10

3.4.3 Cisco AGS+ 10

3.4.4 OSI Echo Function 10

3.5 Routing 10

3.5.1 Intra-Domain Routing Protocol 12

3.5.2 Inter-Domain Routing Protocol 12

3.5.3 Static 12

3.5.4 SunNet OSI 12

3.5.5 IBM OSIMF/6000 13

3.5.6 Cisco AGS +13

4.0 File Transfer, Access and Management 15

4.1 Configuration 15

4.1.1 SunNet OSI 15

4.1.2 IBM OSIMF/6000 16

4.2 User interface 16

4.2.1 SunNet OSI 17

4.2.2 IBM OSIMF/6000 19

4.2.3 Sample Sessions 20

4.3 Interoperability 21

4.4 Performance 21

5.0 FTAM/FTP Application Gateway 23

5.1 User Interface 23

5.1.1 Sample Sessions 24

5.2 Interoperability 26

5.3 Performance 26

6.0 Future Testing 29 


\subsection{Appendix A. OSI Addressing 31}

8.0 Appendix B. The Argonne OSI Addressing Architecture 33

8.1 Background 33

8.2 Alternatives 34

8.3 Argonne Strategy 34

8.4 DECnet Phase V 35

8.5 Internet OSI Activity 36

9.0 Appendix C. Sample Isoentities File 37

10.0 References 41 


\section{LIST OF TABLES}

TABLE 1 GOSIP Versions and Functionality 1

TABLE 2 GOSIP Version 2 NSAP Structure 6

TABLE 3 SunNet OSI FTAM User Commands 17

TABLE 4 SUnNet OSI FTAM Variables 18

TABLE 5 IBM OSIMF/6000 FTAM User Commands 19

TABLE 6 IBM OSIMF/6000 FTAM Variables 20

TABLE 7 FTAM vs. FTP File Transter Times 22

TABLE 8 FTAM vs. FTP Wide Area File Transfer Times 22

TABLE 9 FTP Commands Accepted by the IBM FTAM/FTP Gateway 26

TABLE 10 FTAM/FTP Gateway 'Transfer Times 27

TABLE 11 GOSIP Version 2 NSAP Structure 31 


\section{LIST OF FIGURES}

FIGURE 1 GOSIP Alternatives 5

FIGURE 2 Argonne Testing Environment 7

FIGURE 3 Pouting Standards 11

FIGUFE 4 OS!MF/6000 FTAM/FTP Gateway 23

FIGURE 5 Argonne FTAM/FTP Gateway Testing Environment 24

FIGURE 6 Argonne Intemet Connectivity 33 


\section{Executive Summary}

In the rapidly changing world of communications, remaining current is an ever-challenging objective. At Argonne, we are constantly evaluating and testing new technology as it applies to scientific and administrative computing. Although Open Systems Interconnection (OSI) has been a buzz phrase in the networking community for nearly 10 years, vendors have been slow to bring OSI products to market in the United States, particularly. Now that the U.S. government has mandated the Government Open Systems Interconnection (GOSIP) for all federal computer system procurements, more vendors are marketing GOSIP-compliant OSI products. As new products appear, testing and evaluations need to he conducted to determine the usefulness and applicability of the products in the scientific and administrative computing environment.

At Argonne, we have acquired the SunNet OSI 7.0.1 and IBM OSIMF/6000 Release 1 products for the Sun and IBM RS6000 workstations, respectively. Our experience to date has been that, while we were able to configure and test the products, the ability to manage the products has received little vendor attention. In addition, the human interface to the products needs more attention, and the performance of the products when compared to industry de facto standards such as TCP/IP leaves a lot to be desired. We encourage vendors to continue to enhance their OSI products with better interfaces, better management tools, and better performance as well as more functionality. 


\subsection{Introduction}

The Open Systems Interconnection (OSI) standard is a seven layer reference model that specifies functionality at each layer: the physical, the data link, the network, the transport, the session, the presentation, and (the highest layer) the application layer. Each layer provides specific functions to the layer above it and relies upon specific functions from the layer below. The standard specifies alternatives at most of the layers. If conflicting alternatives are implemented; interoperability may not be achieved. The National Institute for Science and Technology (NIST) holds a number of implementors workshops annually in which industry and government implementors in the United States reach agreement on the way OSI will be implemented on federal computer systems. They thus select a Government OSI Profile (GOSIP), which specifies a subset of the OSI alternatives and their method of implementation.

Congress has amended the Computer Security Act of 1987 to state that all federal computer procurements must comply with any mandatory Federal Information Processing Standards Publications (FIPS PUBS) as specified by NIST. Any FIPS PUBS that bear the word "mandatory" are binding on procurements of federal computer systems, including those procured by DOE contractors such as Argonne. FIPS PUB 146, Government Open Systems Interconnection Profile (GOSIP), is a mandatory FIPS PUB. It states that as of August 15, 1990, GOSIP must be specified when a contractor acquires computer network products and services and communication systems or services that provide equivalent functionality to the protocols defined in the GOSIP documents. Table 1 lists functionality specified in various versions of the GOSIP documents. Additional OSI protocols and services will be included in future versions of the GOSIP FIPS.

TABLE 1 GOSIP Versions and Functionality

GOSIP 1.0

Auqust 1990

File Transfer, Access and Management II

Message Handling System/X.400 1984

Transport Classes 0 and 4

Connectionless Network Service

$X .25$

ISO 8802-3, 8802-4, $8802-5$

\section{GOSIP 2.0}

March 1992

Integrated Services Digital Network

Virtual Terminal Protocol

Office Document Architecture

End System-Intermediate System Protocol

Connection-oriented Network Service

Connectionless Transport Service
GOSIP

Future Versions

File Transfer, Access and Management III

Message Handling System/X.400 1988

Virtual Terminal Protocol extensions

Directory Services

Network Management

Intermediate System -Intermediate System Protocol 
Argonne has a large, heterogeneous networking environment that serves scientific, engineering, and administrative staff in a campus setting. The Laboratory's strategy for support of computer networks is based on local area networks (LANs) that encompass buildings and programs, a Laboratory-wide network to interconnect LANs, and gateways between the Laboratory-wide network and various national and international networks. Argonne's current networks are based on the following protocols: IBM's SNA, TCP/IP, DECnet, XNS, AppleTalk, and IPX. The Laboratory's LANs are mainly Ethernet-based networks. The Laboratory-wide network is FDDI-based. External network connections include ESnet, HEPnet, CICNet, NSFnet and BITnet. Connectivity to the onsite as well as external networks is available from a wide variety of over 1,800 workstations and personal computers (Sun workstations, Apple Macintoshes, IBM PCs, etc.), mid-level computers (DEC VAXes), mainframe computers (Cray XMP and IBM 3084), and a variety of experimental parallel processors (Sequent, Thinking Machines Connection Machine, etc.).

The future direction of networking at Argonne is being planned with GOSIP as a focus, although GOSIP products are just becoming available and the standards are still in the process of being interpreted and amended. Our goal is to use GOSIP protocols to achieve interoperability with other DOE sites by October 1995. Acceptable strategies for implementing GOSIP include the use of dual-protocol hosts and application layer gateways. Argonne is pursuing alternatives to achieve GOSIP compllance without adversely affecting the networking needs of the Laboratory's scientific, engineering, and administrative staff and, at the same time, is interested in protecting the Laboratory's investment in computer network products and services, and communications systems and services.

One alternative we are focusing attention on is the use of application layer gateways between the protocols commonly used at Argonne and GOSIP protocols. It is our goal that the GOSIP transition strategy we develop may be used by other sites in developing their own strategy. The findings we have presented in this document will form the basis for a GOSIP transition strategy for Argonne.

This document describes testing of OSI products conducted at Argonne National Laboratory. Sun, IBM, and Clsco hardware platforms were used. Various software packages that implement file transfer and gateway applications were evaluated. The OSI model and GOSIP compliance are briefly discussed. Technical details on OSI addressing and routing are presented. The relationship of this testing to other OSI activities at Argonne and to activities of the national networking community is discussed. Mention is also made of the relationship of DECnet Phase $V$ transition issues. 


\subsection{The GOSIP Testing Environment at Argonne}

Because of the complcx variety of alternatives available to implement GOSIP-compliant networking environments, it is necessary to establish technical studies and prototype networks to evaluate newly emerging GOSIP products. Testing GOSIP products can be accomplished at different levels--conformance testing to show that the vendor has met the GOSIP protocol specifications, interoperability testing to prove that hardware and software components can interoperate (not simply coexist), performance testing to show that products perform required functions adequately, user interface testing to evaluate the usability of the human interface, and operational testing to determine the manageability of the software. Argonne's interest lies in the areas of interoperability testing, performance testing, user interface evaluation, and operational testing.

The hardware platforms on which the tests were performed during the initial phases of this project include a Sun Sparcstation 1t, an IBM RS6000 Model 520, and a Cisco AGS+ router. Software installed on the Sparcstation is Sun's SunNet OSI 7.0.1 and SunNet MHS 7.0. Software installed on the RS6000 is IBM's OSI Messaging and Filing/6000 (OSIMF/6000) Release 1. Testing between these systems was performed via a 802.3 local area network. Additional testing was performed over the ESnet-wide area network between two Sun Sparcstation 1+s, both running SunNet OSI 7.0.1. The router platform selected for all of Argonne's wide-area connectivity is the Cisco Systems, Inc. AGS+ running software Release 8.2(7).

While individual site transition strategies are important, interoperability among sites requires extensive coordination and interoperability testing. Argonne has been working with various members of the Internet community to test OSI interoperability over a wide-area network. We have participated on the ESnet OSI Routing Task Force to test and document a strategy for routing OSI traffic over ESnet and have participated on the ESnet X.400/X.500 Task Force to define a strategy for X.400 and X.500 implementation on ESnet. Argonne has worked with $\mathrm{CICNet}$ to implement OSI routing to CICNet member institutions in the midwest. Extensive wide-area OSI interoperability testing over the Internet was conducted on about 30 networks (including Argonne, CICNet, ESnet and NSFnet) during the summer of 1991, culminating in an OSI InterOp '91 demonstration in October 1991. This testing was orchestrated by the NSFnet staff at Merit Network, Inc. Argonne's participation included implementing OSI routing, FTAM interoperability testing, and demonstration of FTAM and X.500. 


\subsection{Network Services}

In OSI terminology, a subnetwork is what was previously considered to be a single network (e.g., several nodes connected together on a single transmission medium). An end system (ES) is a node in the network containing all seven layers (a host). An intermediate system (IS) is a single node, connected to more than one subnetwork, where the IS can forward data from one subnetwork to another (a router). An IS is not required if all ESs are connected to a single subnetwork; however, it is recommended because it avoids the need to maintain static address tables. An IS need only have a subset of the OSI layers present, namely layers 1 through 3. At the simplest level, a "network" is composed of a collection of subnetworks, connected by ISs and populated by ESs.

The network service layer is responsible for moving data from one system to another. Unfortunately, in OSI, two network services are specified, and they are largely incompatible: connection-oriented (CO) [1] and connectionless (CL)[2].

\subsection{GOSIP Alternatives}

Figure 1 illustrates the various options available at layers 1 through 4 under GOSIP Version 1. CL-mode Network Service (CLNS) and transport class 4 are mandated by GOSIP for connecting ESs to subnetworks. CO-mode Network Service (CONS) is an option available to GOSIP ESs directly connected to an X.25 subnetwork. CONS and transport class 0 may be used in conjunction with message handling service $(X .400)$ via the public data network.

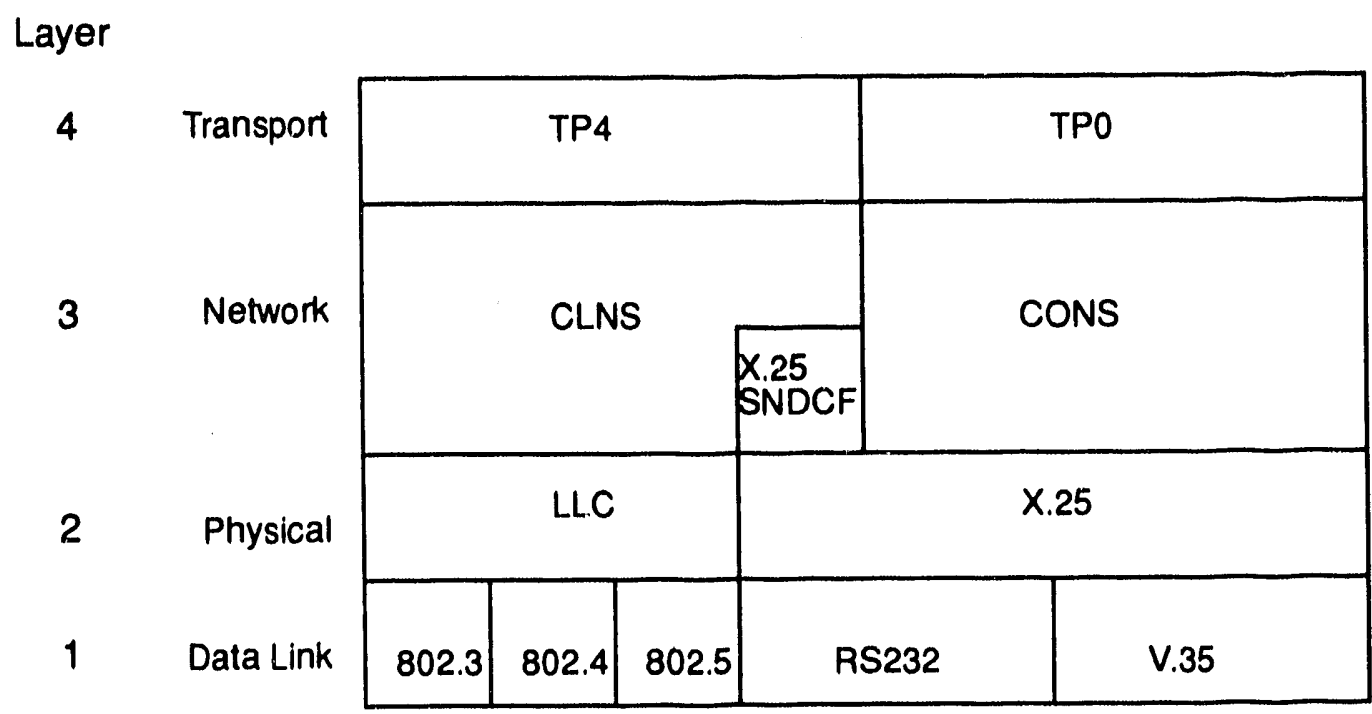

FIGURE 1

GOSIP Alternatives

The GOSIP alternatives are likely to be expanded in the future. GOSIP Version 1 compliance is mandated as of August 15, 1990. It is legitimate (but not mandatory) to use technology specified in a future version of GOSIP (e.g., GOSIP Version 2), once the feature is announced. For example, a site 
may implement the ES-IS protocol specified in GOSIP Version 2 and still te GOSIP-compliant, assuming the site also meets the other GOSIP Version 1 requirements.

\subsection{OSI Addressing}

An OSI system address consists of an Network Service Access Point (NSAP) address plus the selectors required to reach application processes. Selectors are used to direct packets to specific entities within a system as the packet moves upward through successivs layers toward a destination application process. A selector identifies an entity in the layer above. ISO 8348/Addendum 2 [2] defines the semantics of network layer addressing. GOSIP Version 1 Secticn 5.1.1 was amended to align with the GOSIP Version 2 NSAP address format, which is depicted in Table 2. For a more detalied description of the GOSIP NSAP structure, refer to Appendix A.

TABLE 2 GOSIP Version 2 NSAP Structure

\begin{tabular}{|c|c|c|c|c|c|c|c|c|c|}
\hline \multirow{3}{*}{$\begin{array}{l}\text { ISONSAP } \\
\text { S Governmont IDI }\end{array}$} & \multicolumn{2}{|c|}{ IDP } & \multicolumn{5}{|c|}{ HODSP } & & \\
\hline & AFI & $|D|$ & & & & & & & \\
\hline & 47 & 0005 & DFI & AA & Res & RD & Area & Sijstem ID & NSEL \\
\hline octets & 1 & 2 & 1 & 3 & 2 & 2 & 2 & 6 & 1 \\
\hline
\end{tabular}

\footnotetext{
IDP - Initial Domain Part HO-DSP - Hlgh-Order Domain Specific Part

AFI - Authority and Format Identifier

IDI - Initial Domain Identifier DSP - Domain Spocific Part DFI - DSP Format Identifier

AA - Administrative Authority ino - raserved for future use RD. Routing Domain

System ID - System Identifier

NSEL - NSAP Selector
}

Argonne has been assigned the Routing Domain value 0010 (hexadecimal) from the ESnet Administrative Authority 005700 (hexadecimal). Included in Appendix B is an explanation of how Argorine arrived at its NSAP address prefix. At Argonne, the first six fields of the NSAP address (prefix) are constant (47.0005.80.005700.0000.0010). The Area field is assigned based on campus location. The System ID is the MAC address (Ethernet hardware address) of the system. NSAP addresses for the Argonne ESs are:

- Sun ES 47.0005.80.005700.0000.0010.c000.080020090576.00

- IBM ES 47.0005.80.005700.0000.0010.c000.02608c2fe9f5.01 
A Network Entity Title (NET) is the single network layer address by which an IS is known on all subnetworks to which it is connected. An NET takes the form of an NSAP address, minus the NSEL. NET addresses for the Argonne ISs are:

- Cisco IS 47.0005.80.005700.0000.0010.c000.00000c0136de

- Cisco IS 47.0005.80.005700.0000.0010.0001.aa00040057ba

- Cisco IS 47.0005.80.005700.0000.0010.0001.000000000001

Figure 2 shows the Argonne environinent in which the testing described in this document was performed.

To communicate with an application on another ES, the initiating host must know the presentation address (the NSAP address plus the selectors) required to reach the application process running on the destination machine. A presentation address consists of the NSAP address, a transport selector (TSEL), and optional session and presentation selectors (SSEL, PSEL). The presentation address must be available for the application initiator to contact the remote application. Ideally, the presentation address and host name mapping will be stored and available through X.500 Directory Services. Today, however, this information is stored in static host tables.

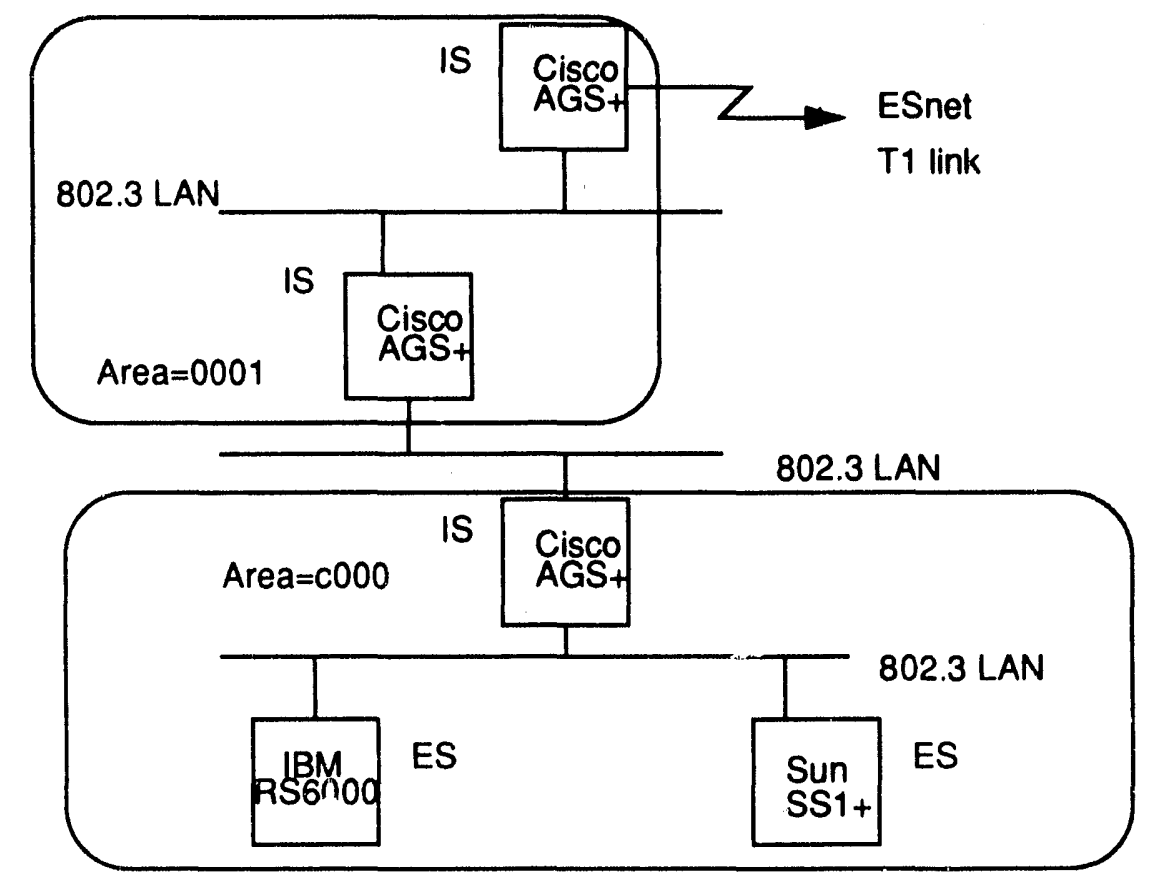

FIGURE 2

Argonne Testing Environment 


\subsection{Configuration}

In this section we describe the process a systems administrator must complete to configure the network service layer of each of the products we evaluated. Along with each description, we have described the configuration used at Argonne, along with our comments on each process.

\subsubsection{SunNet OSI}

SunNet OSI provides a variety of subnetwork connection options: X.25, LLC1 over 802.3 or FDDI, and LLC2 over 802.3. The SunNet OSI product implements the following transport stacks: RFC1006 over TCP/IP, TPO or TP2 over X.25, and TP4 over CLNP. A single machine may be configured to run any or all of these options. To run over X.25, an additional Sun product, SunNet X.25 is required. Argonne has not experimented with the X.25 product. SunNet OSI recognizes and handles addresses conforming to ISO 8348/Addendum 2 [2]. At Argonne we configure SunNet OSI to be GOSIP-compliant using LLC1 over 802.3 and TP4 over CLNP.

SunNet OSI is shipped with an install.osi script, which prompts the system administrator for the options necessary to configure the software. As a result, it produces a few configuration files of interest:

- /etc/sunlink/osi/routes--file containing CLNP and ES-IS routing information read by the SunNet OSI routing daemon, osi.routed

- letc/sunlink/osi/rc--script used to start SunNet OSI

- /etc/sunlink/osi/isoentities--file containing remote responder applications for local applications running over SunNet OSI

The isoentixies file performs the function of a directory service. Local initiator applications use entries from isoentities to locate the presentation address of remote responder applications. Each entry in isoentities contains:

- hostname--identifies a host on the OSI network

- application name or service--identifies the service to be provided by the remote host

- application entity title--the ISO unique identifier for the application process

- presentation address--complete address required to reach the remote application

A sample isoentities file appears in Appendix C. A utility (entities.add) is provided with the package to provide a friendly method for adding entries to the isoentities file. The file can be edited and viewed with standard Unix text editing tools (i.e., vi, ernacs).

The start-up script, rc, will initiate three Unix processes:

- tsapd--starts up responder applications

- osi.routed--performs CLNS and ES-IS protocol

- osi_pingd--responds to ISO Echo packets 
The SunNet OSI product is well-documented, and the installation procedures supplied make installation straightforward.

\subsubsection{IBM OSIMF/6000}

OSIMF/6000 may be configured for up to two of the following subnetwork connection types: 802.3 , 802.5 , or X.25. OSIMF/6000 implements the following transport classes: TPO or TP2 over CONS or TP4 over CLNS. At Argonne, we configure OS!MF/6000 to be GOSIP-compliant using 802.3, and TP4 over CLNS. All configuration for the OSI protocol stack layers 3 through 6 is done by the system administrator in the file /etc/osi.cfg. IBM does not provide tools to update or validate the entries in the configuration file. We did contact one of the developers from IBM Dallas who had written such a utility, but IBM does not distribute it with the OSIMF/6000 software. Such a utility is essential for the novice administrator. The /etc/osi.cfg file is very intimidating. IBM's documentation on the setting of the parameters in /etc/osi.cfg needs improvement.

OSIMF/6000 uses the same format isoentities file described under SunNet OSI to perform the directory service function. IBM does not provide a utility to add entries to the file. Standard AIX utilities must be used to update isoentities.

The OSI protocol stack and applications are started and stopped with the /etc/rc.osimf script. We have experienced problems stopping or unloading the OSI stack on the RS6000. On occasion, stopping the process has caused AIX on the RS6000 to crash. The problem has been reported to IBM, but no fix has been provided.

\subsubsection{Clsco AGS+}

Cisco meets ISO standards 8473 [9] and 8348/AD2 [2] for CLNS and network layer addressing, respectively. Configuration of a Cisco IS involves setting a NET address, enabling CLNS routing on the necessary interfaces, and setting up the routing configuration to use either static or dynamic routir:g (see "Routing" section).

\subsection{Management}

All of the products evaluated in this study suffer from a common flaw. None of the vendors has implemented the Virtual Terminal protocol in its products; therefore, it is necessary to use the TCP/IP Telnet protocol to communicate with the remote machine if the machines are not collocated.

\subsubsection{SunNet OSI}

SunNet OSI offers several diagnostic tools to enable systern administrators to troubleshoot problems:

- osi_ping--sends a CLNP echo packet to a remote host to determine if a route is available between the local machine and the remote machine

- osirstat--displays the current state of the OSI routing tables

- osi_trace--displays packets on Ethernet or FDDI subnetworks 


\section{Network Services}

Sun has done a nice job of providing tools within the OSI environment similar in function to those provided in the TCP/IP environment. The lack of standards for such tools, however, has hindered the usefulness of these tools among systems of different vendors.

\subsubsection{IBM OSIMF/6000}

OSIMF/6000 lacks tools for administration of the ES. This is an severe shortcoming of this package. At a minimum, a method is needed to dump the routing table of the ES and to send and receive echo packets.

\subsubsection{Clsco AGS+}

Cisco provides the following CLNS diagnostic tools:

- CLNS trace--traces a packets route through a CLNS network by sending probe packets taking advantage of the error report protocol data units (PDUs) generated when a packet exceeds its time-to-live value

- CLNS ping--sends echo PDUs to test whether a destination is reachable

- show CLNS route--displays all of the destinations to which the IS knows how to route packets

- show CLNS neighbors--displays both ES and IS neighbors of the IS

Early versions of the Cisco CLNS software contained a severe bug that caused a router to crash when the CLNS ping command was used by the Cisco administrator. This problem has been corrected in Release 8.2(7) of the Cisco software.

\subsubsection{OSI Echo Function}

ISO 8473 [9] defines a means for transmitting and relaying data and error report PDUs through an OSI network. Unfortunately, the network through which these packets travel is imperfect. Compo. nents will fail. An echo function is imperative in debugging and testing the OSI network layer. A simple mechanism in the network layer is required so that systems can be probed to determine if the lowest levels of the network software are operating correctly. Request for Comment (RFC) 1139 [15] defines a standard OSI echo function for hosts connected to the Internet.

SunNet OSI has implemented an RFC1139 compliant echo function; however, Cisco and IBM have not. Therefore, the SunNet OSI osi_ping function is only useful between Sun systems. The CLNS ping function provided by Cisco is only useful between Cisco ISs. IBM has not implemented any echo function. The echo function is an essential tool for network administration and debugging. A standard method of implementation is needed, and all OSI vendors should include this function in their products.

\subsection{Routing}

The basic principles of Network Layer routing are defined in the OSI Routeing Framework, TR9575 [3]. GOSIP Version 1 requires the implementation of static CLNP routing. Static routing, however, is 
not sufficient for most environments. The global OSI environment will consist of a number of administrative domains. An administrative domain is a collection of ESs, ISs, and subnetworks operated by a single organization or administrative authority (AA). The AA is responsible for the organization of ESs and ISs into routing domains, the policies that govern resource usage, the policies that govern information that is collected and disseminated both internally and externally to the administrative domain, and the establishment of subdomains and the corresponding delegation of responsibilities. A routing domain is a set of ESs and ISs that operates according to the same routing procedures and that resides within a single administrative domain. The decomposition of routing domains into subdomains (areas) may greatly reduce the resources required to maintain, compute, and store routing information. Figure 3 illustrates the routing principles described in the following text.

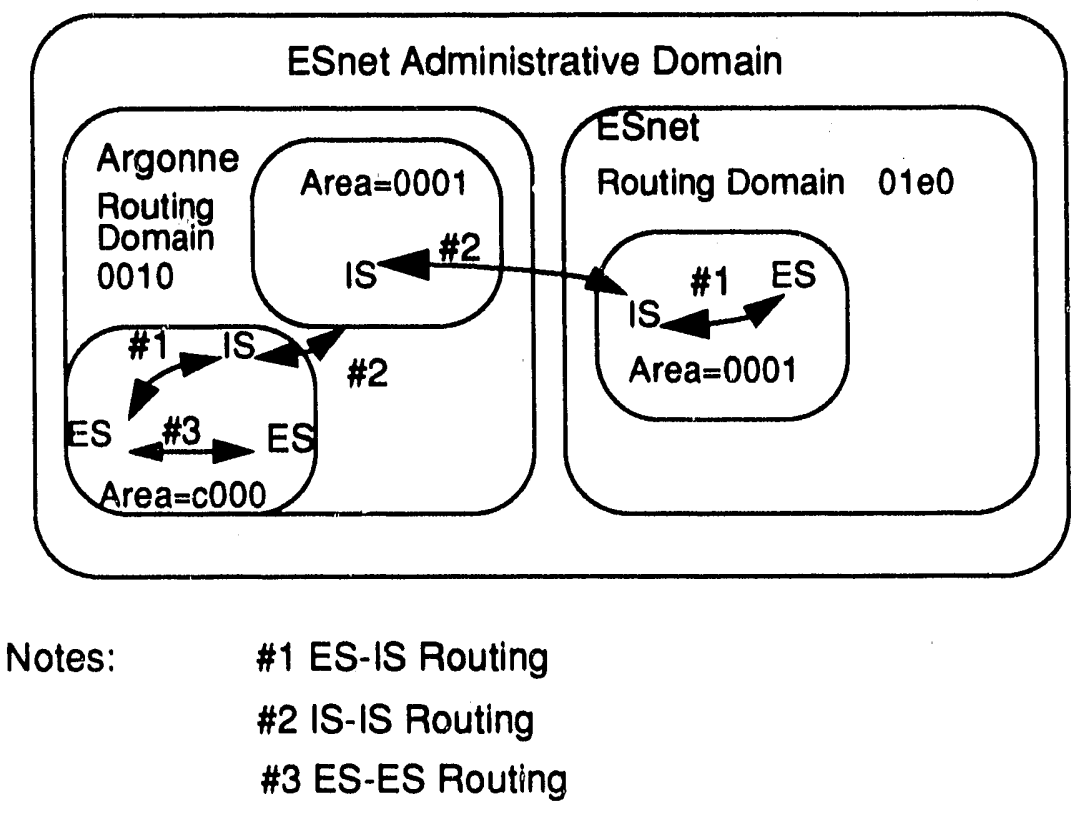

FIGURE 3 Routing Standards

Argonne has elected to participate in the ESnet administrative domain; therefore, Argonne has been assigned a RD from the ESnet AA space. Within its RD, Argonne is free to assign areas and system ids. Routing exchanges between the Argonne RD and any other AA must be coordinated through ESnet.

The practicality of deploying dynamic, adaptive routing protocols in large production networks depends on the commercial availability of mature, interoperable products that conform to the appropriate international standards and implementors agreements. Today, the reality is that these products are not readily available. NIST is coordinating an OSI Routing Laboratory which will test the interoperability of vendors routing products. The testing will continue in calendar year 1992. 


\subsubsection{Intra-Domain Routing Protocol}

To handle large routing domains, intra-domain routing is organized hierarchically. A large routing domain may be administratively divided into areas. Routing within an area is referred to as Level 1 routing. Routing between areas is referred to as Level 2 routing. Level 2 ISs keep track of the paths to other areas. Level 1 ISs keep track of the routing within their own area. End-system-to-intermediate-system (ES-IS) routing ivithin a single subnetwork is defined by ISO 9542 [6]. Each system (ESs and ISs) lives in one area. Each ES must be able to communicate with a Level 1 is in its area. An ES will discover the nearest Level 1 IS by listening for ES-IS hello packets broadcast on the subnetwork.

When an ES has a packet to send to another ES, it sends it to the Level 1 IS for its area. The IS will look up the destination NSAP and forward the packet along the best route. If the destination NSAP specifies an ES in another area, the Level 1 IS will send the packet to the nearest Level 2 IS within the area. The Level 2 IS will forward the packet along the best path for the destination area until it reaches a Level 2 IS in the destination area. This IS will forward the packet along the best path (through Level 1 ISs) within the area until it is delivered to the destination ES.

The IS-IS standard defines the protocol by which ISs exchange routing information. The IS-IS standard has progressed to Draft Proposal status [8]; however, not all vendors have implemented IS-IS functionality.

\subsubsection{Inter-Domain Routing Protocol}

The Inter-domain Routing Protocol (IDRP) specifies a protocol to be used by boundary ISs to maintain information about the interconnections between routing domains. At this time, IDRP has not advanced in the standards track and remains a Draft International Standard at this time. Only early experimental versions of the protocol are in the field. Until the standard advances and vendors commit to implementation, IDRP will not be 2 viable option for inter-domain routing. IDRP is essential in the global OSI Internet. It will be used to exchange routing information between administrative domains (e.g., the National Science Foundation Network, the Energy Sciences Network, and the NASA Science Internet).

\subsubsection{Static}

Static routing is used when it is not possible or desirable to use dynamic routing. The consequences of static routing are that there is no adaptive routing around failed components, it is difficult to administer in the long run, and load sharing is not possible. Static routes are entered by specifying pairs of addresses: NSAP-prefix and next-hop-NET. Implementations that perform static routing only will not be useful in the global OSI Internet. They may be useful in a localized OSI network.

\subsubsection{SunNet OSI}

SunNet OSI implements and performs static routing as well as the ES and IS functions of the ES-IS protocol. Therefore, a Sun may act as an is to forward packets between OSI subnetworks. The ES-IS protocol provides dynamic routing information on the OSI subnetwork. Argonne uses the ES 
functions of the ES-IS protocol on the Sun. The ES-IS protocol is well-documented and easy to implement. We recommend the use of ES-IS protocol to avoid the need to maintain static tables.

\subsubsection{IBM OSIMF/6000}

OSIMF/6000 can use static routing information and/or dynamic routing information. IBM implements and supports only the ES functions of the ES-IS protocol. Therefore, the RS6000 cannot forward packets between subnetworks for other ESs. We recommend the use of the ES-IS protocol. The IBM documentation does a poor job of explaining how to use the ES-IS protocol, and configuring it is not straightforward.

\subsubsection{Clsco AGS+}

Cisco implements static and ISO 9542 [6] ES-IS routing. For dynamic routing with ISO CLNS, Cisco implements the Interior Gateway Routing Protocol (IGRP). This protocol is a Cisco proprietary protocol, based on the IGRP protocol for IP networks, which can only be used between Cisco routers. At this time, Cisco's IGRP provides a useful alternative to static routing; however, the long-term solution is the adoption of standard protocols IS-IS and IDRP. We expect that Release 9 of the Cisco software (to be available around spring 1992) will implement IS-IS. Clsco recognizes the importance of IDRP but, at this time, has not committed to an implementation date. 


\subsection{File Transfer, Access and Management}

File Transfer, Access and Management (FTAM) provides the fille service for OSI. It is important to recognize that FTAM, defined in ISO 8571 [14], is more than a file transfer facility. The cornerstone of FTAM is the virtual filestore that describes a conceptual file service model to provide an abstract capable of being emulated on top on the file services offered by any computer. A virtual filestore is a collection of files. There is currently no explicit relationship among files in the filestore. FTAM may be appropriate for filestore-to-filestore transfer, diskless workstation file access, special applications such as printing and spooling, and remote database access. The SunNet OSI and OSIMF/6000 preducts are limited in scope to the file transfer application. In addition to the file transfer application, the IBMI OSIMF/6000 product includes a FTAM/FTP application gateway.

GOS:P Version 1 includes the following FTAM document types (file structures):

- FTAM-1 Unstructured Text

- FTAM-2 Sequential Text

- FTAM-3 Unstructured Binary

- NBS-4 Sequential Binary

- NBS-5 Simple Hierarchical

- NBS-6 Sequential

- NBS-7 Random Access

- NBS-8 Indexed

- NBS-9 File Directory

GOSIP Version 1 requires implementation of document types FTAM-1 and FTAM-3 only.

\subsection{Configuration}

\subsubsection{SunNet OSI}

SunNet OSI is shipped with the FTAM initiator (client) and responder (server) programs. For the FTAM responder, SunNet OSI uses selector values: TSEL \#259 (decimal), PSEL NULL, and SSEL NULL. The FTAM responder program maps all virtual filestore operations to SunOS file operations. Sun supports three FTAM document types: FTAM-1, FTAM-3, and NBS-9. Before an FTAM association can be established with a remote host (responder), the presentation address of the remote host must be entered in /etc/isoentities file.

The FTAM responder program is set up to run automatically when SunNet OSI is started. The dynamic daemon, ftamd, is started on demand by the tsapd daemon. For authentication, the user must supply a valid username and password on the Sun server to establish a connection with the SunNet OSI responder. The SunNet OSI responder also supports anonymous FTAM access as described below (see "User Interface" section). 


\subsubsection{IBM OSIMF/6000}

OSIMF/6000 is shipped with the FTAM initlator (client), FTAM responder (server), and FTAM/FTP gateway programis. For the FTAM responder, OSIMF/6000 uses selector values: SEL \#259 (decimal), PSEL NULL, and SSEL NULLL. OSIMF/6000 supports three FTAM document types: FTAM-1, FTAM-3, OSIMF (IBM's File Directory). Before an FTAM association can be established with a remote host (responder) the presentation address of the remote host must be entered in /etc/isoentities file.

The system administrator starts the FTAM responder application and FTAM/FTP gateway by issuing the command rc.osimf -ftam.

\subsection{User Interface}

The FTAM initiator program (client) allows a user to perform operations on files residing on a remote server that is running the FTAM responder program (server). An FTAM responder requires the user to provide a valid username and password for the target host before allowing files to be manipulated. An FTAM responder may provide an account that may be used by any initiating user. This FTAM account, commonly referred to as an ANONYMOUS account, is accessed by providing the user identity ANONYMOUS and any password. The password is not validated. Since any user can use this account, it may be considered a security risk. While the anonymous access method is inherently risky, it is also inherently useful.

The user may create the ftamrc file in his home directory that includes FTAM commands to be executed every time FTAM is invoked. Many of the FTAM commands may be entered in interactive mode or on the command line. 


\subsubsection{SunNet OS:}

The Sun FTAM initiator has a user interface modeled on the interface to the SunOS TCP/IP file transfer program (FTP), which is a standard part of the Sun operating system. Table 3 lists the user commands avallable with the SUnNet OSI FTAM initiator program.

TABLE 3 SunNet OSI FTAM User Commands

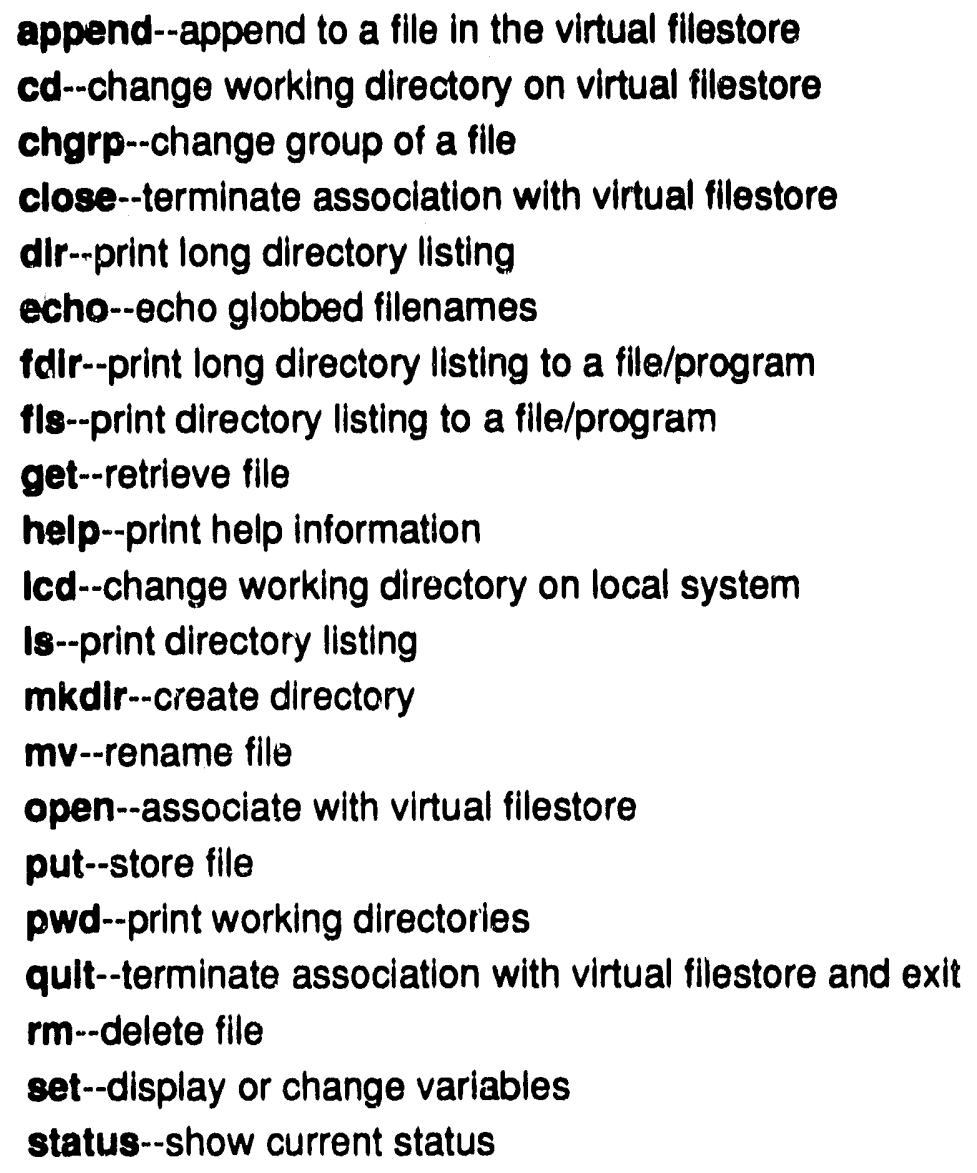

Table 4 lists the variables that affect FTAM operations. The user may set the variables by using the FTAM "set" command. 
TABLE 4 SunNet OSI FTAM Variables

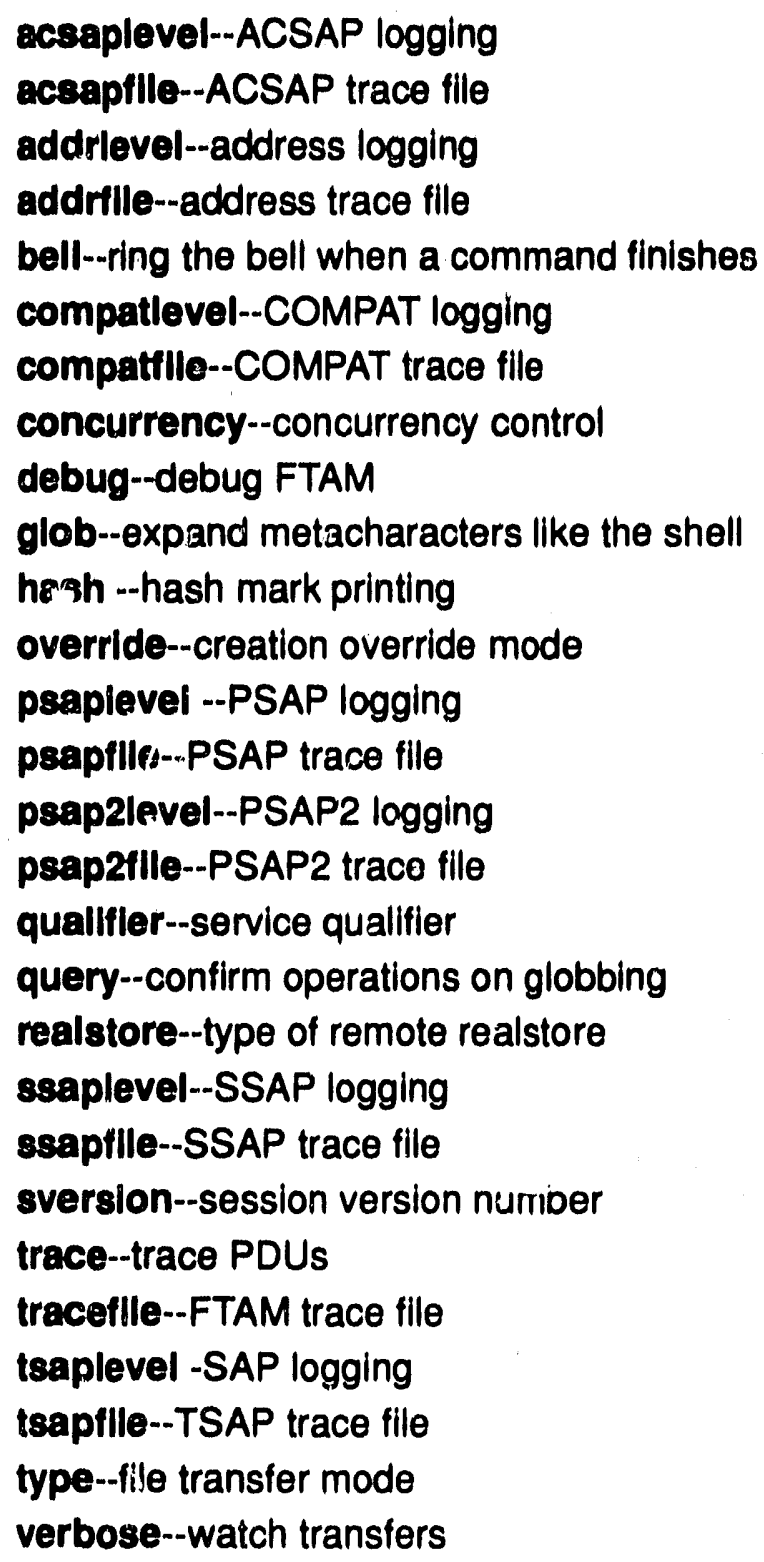

We found the error messages produced by FTAM to be unintelligible to the average user. The SunNet OSI FTAM User's Guide[13] needs to include descriptions of the likely causes of the errors. 


\subsubsection{IBM OSIMF/6000}

The IBM FTAM initlator has a user interface modeled on the interface to the AIX file transfer program (FTP). Table 5 lists the user commands avallable with the IBM OSIMF/6000 FTAM initiator.

TABLE $5 \quad$ IBM OSIMF/6000 FTAM User Commands

append--append to a fille in the virtual fllestore chgrp--change the group of a file in the virtual filestore

close--terminate the association with a virtual filestore echo--echo globbed filenames

get--transfer a file from the virtual filestore help--display information about FTAM commands and variables led--change the current working directory on the local system mv--rename a file in the virtual filestore open--associate with a virtual filestore put--transfer a file to the virtual filestore pwd-print the current working directory on the local system quit--terminate the current association and exit rdattr--read attributes of the remote file rm--delete a file from the virtual filestore set--display or change variables status--show the status of the current association

Table 6 lists the FTAM variables that affect IBM OSIMF/6000 FTAM operations. The user can set the variables by using the FTAM "set" command. 
TABLE 6

IBM OSIMF/6000 FTAM Variables

bell--rings the bell when a command is completed

hash--activates display of the quantity of data transferred during the FTAM file transfer

override--sets the creation override mode for files created on the remote filestore

qualifier--sets the qualifier for the FTAM server

query--activates queries to the user to confirm operations involving expressions that expand to more than one filename

realstore-informs OSIMF/FTAM whether or not the server operating system is Unix-based trace--activates logging of PDUs exchanged between client and server during an FTAM session

traceflle--specifies the file where trace records are to be stored

type--defines the FTAM file type of the files to be transferred

verbose--displays detailed descriptions and feedback about FTAM commanci execution watch--displays additional feedback as FTAM operations are performed

A severe limitation of the OSIMF/6000 user interface is the lack of the Is (print directory listing) and cod (change directory) commands. Users must know the full path and filename of the file they wish to transfer. The name of the file is very often not known and the directory list and change directory commands are essential. Because IBM has not implemented the standard document type NBS-9, issuing the print directory listing command from another vendor's initiator to an IBM repsonder is useless. The goal of FTAM is standardization; however, IBM has strayed from the standard by defining its own document type for the directory. We f ind the error messages produced by the FTAM initiator to be unintelligible to the average user. IBM needs to provide more meaningful error messages and include documentation of the messages in the user manual [10].

\subsubsection{Sample Sessions}

In this section, sample FTAM sessions are illustrated. In all examples, the boldface type represents system responses. The user input is shown in normai typeface. In the first example, the user initiates FTAM on host "hamlet" (IBM) by opening an association with machine "olivia" (Sun), logs on to "olivia" as "test," and then uses the "put" comınand to transfer file BIG1 before exiting the FTAM program.

hamlet\% ftam olivia

user (ollvla:osidemo): test

password (olivia:test):

olivia... connected

olivias put BIG1

destination: blg1

1095022 bytes transferred in 21.34 seconds (50.11 Kbytes/s)

oliviasquit 
In the following example, the user initiates FTAM on machine "olivia" (Sun), sets the concurrency variable off, opens an association with machine "hamlet" (IBM), logs on to "hamlet" as user "test," and uses the "put" command to transfer file BIG1 before exiting the FTAM program.

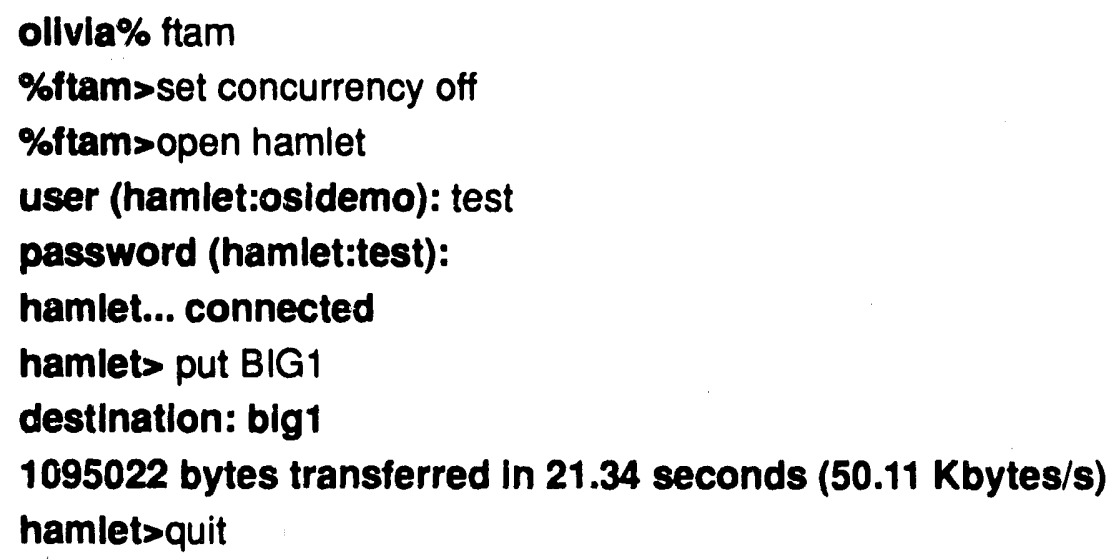

\subsection{Interoperability}

The OSIMF/6000 FTAM initiator can send (put) and retrieve (get) files from the SunNet OSI responder without problem. We did encounter difficulty in two areas, however, when the SunNet OSI initiator attempted to communicate with the OSIMF/6000 responder. Sun has implemented concurrency control in the FTAM initiator. When the user attempted to transfer a file between the SunNet OSI initiator and OSIMF/6000 responder, the message "Concurrency control not supported" appeared if the user failed to set concurrency off. The concurrency control function indicates access available to the user and access available to other users. This is an optional FTAM function. For the Sun initiator and IBM responder to interoperate, enter the "set concurrency off" command before opening the FTAM connection as illustrated in the above sample session. The second problem occurred when attempting to transfer a file in binary mode. The Sun FTAM initiator required the file to be a binary file. If the file contained only ASCII text, the message "Subsequent error type transient" appeared. The solution to this problem is to set the FTAM type variable to the correct type (default, binary or text) for the file to be transferred.

\subsection{Performance}

To measure the performance of the FTAM impiementations, a sample file $(1,093,384$ bytes) was transferred between the Sun and IBM machines over a local area network. As a means of comparison, the same file was also transferred between the same machines via the TCP/IP file transfer program (FTP). The results are summarized in Table 7. In the table, "IBM->Sun" indicates the IBM initiated the transfer with the Sun responder and "Sun->|BM" indicates the Sun initiated the transfer with the IBM responder. In the case of the lines labeled "Sun->Sun" and "IBM->IBM," the transfers were performed entirely within the same machine. 
TABLE 7 FTAM vs. FTP File Transfer Times (Kilobytes/second)

\begin{tabular}{|c|c|c|c|c|}
\hline Transfer Type & FTAM & FTAM & FTP & FTP \\
\hline File Type & binary & ascii & binary & ascii \\
\hline IBM->Sun (get) & 139.9 & 63.7 & 495.7 & 140.1 \\
\hline IBM->Sun (put) & 80.9 & 71.7 & 204.9 & 130.6 \\
\hline IBM->IBM (get) & 42.4 & 37.3 & 744.9 & 58.7 \\
\hline IBM->IBM (put) & 57.9 & 71.7 & 647.2 & 75.5 \\
\hline Sun->IBM (get) & 102.9 & 67.5 & 197.5 & 118.7 \\
\hline Sun->IBM (put) & 160.0 & 91.0 & 475.0 & 121.3 \\
\hline Sun->Sun (get) & 219.3 & 71.3 & 712.5 & 72.6 \\
\hline Sun->Sun (put) & 225.5 & 74.4 & 745.0 & 73.3 \\
\hline
\end{tabular}

Additional testing was performed over the ESnet wide area network between two Sun Sparcstation $1+s$, both running SunNet OSI 7.0.1. Table 8 summarizes the timings of the test performed over the wide area network. In Table 8, "Sun->Sun" indicates the transfer was between one Sun located at Argonne and another Sun located at Lawrence Livermore National Laboratory. Our conclusion from these tests is that the FTAM transfer application is significantly slower than FTP, particularly in the case of the binary transfer. We encourage vendors to enhance the performance of the FTAM application.

TABLE 8 FTAM vs. FTP Wide Area File Transfer Times (Kilobytes/second)

$\begin{array}{lcccc}\text { Transfer Type } & \text { FTAM } & \text { FTAM } & \text { FTP } & \text { FTP } \\ \text { File Type } & \text { binary } & \frac{1}{\text { ascii }} & \text { binary } & \text { ascii } \\ \text { IBM->Sun (get) } & 6.5 & 5.7 & 33.4 & 31.9 \\ \text { IBM->Sun (put) } & 24.6 & 23.9 & 15.6 & 16.4 \\ \text { Sun->IBM (get) } & 22.4 & 23.1 & 15.6 & 16.8 \\ \text { Sun->IBM (put) } & 7.9 & 7.4 & 34.5 & 32.3 \\ \text { Sun->Sun (get) } & 56.5 & 38.8 & 38.6 & 39.6 \\ \text { Sun->Sun (put) } & 14.6 & 11.6 & 18.3 & 18.1\end{array}$




\subsection{FTAM/FTP Application Gateway}

The FTAMFTP application gateway, supplied as a component of the IBM OSIMF/6000 product, connects users of FTAM with users of TCP/IP FTP, providing bidirectional ASCII and binary file transfer. On the FTAM-to-FTP side, the FTAM/FTP gateway translates FTAM file transfer commands into requests recognized by FTP servers. On the FTP-to-FTAM side, the FTAM/FTP gateway translates FTP commands into requests recognized by FTAM servers. Figure 4 illustrates the path the transfers take via the FTAM/FTP gateway.

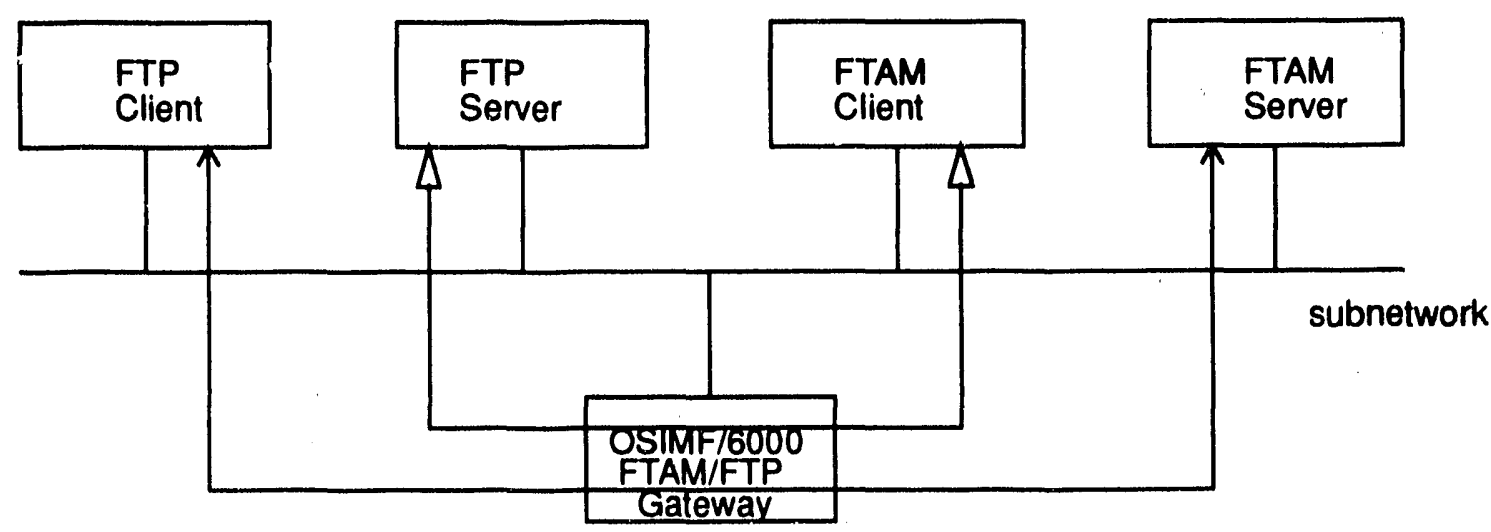

FIGURE 4 OSIMF/6000 FTAM/FTP Gateway

\subsection{User Interface}

Use of the FTAM/FTP gateway is not transparent. The user must know the location of the gateway and the special sequence of commands required to communicate via the gateway. This is a limitation which will make it painful for the user to make use of the gateway's functionality. Figure 5 illustrates the Argonne test environment for the FTAM/FTP gateway. 


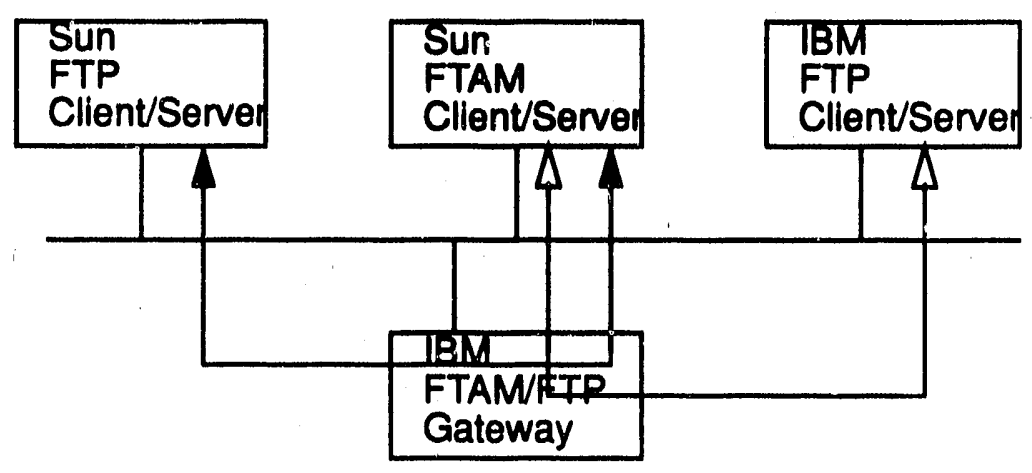

FIGURE 5 Argonne FTAM/FTP Gateway Testing Environment

\subsubsection{Sample Sesslons}

The following is a sample of a Sun FTP client to Sun FTAM server session via the OSIMF/6000 FTAMFTP gateway. First, the user must FTP to port 562 on the machine supplying the FTAM/FTP gateway ("hamlet.ctd.anl.gov" in this case). When prompted for a name, the user responds with the userid and hostname of the target FTAM server. Once the connection is established by the gateway, the user may enter valid FTP commands, which the gateway then translates to the appropriate 
FTAM equivalent commands and sends to the remote FTAM server. In all the examples, system responses are shown in boldface type and strings entered by the user are shown in normal typeface.

achilles.ctd.anl.gov\%ftp hamlet.ctd.anl.gov 562

Connected to hamlet.ctd.anl.gov.

220 hamlet FTP/FTAM gateway (Version 4.89 Tue Jun 25 22:11:20 PDT 1991) ready.

Name (hamlet.ctd.anl.gov:b32357): b32357@olivia

331 Password required for "b32357@olivia".

Password:

200 Logged Into ollvia

ftp> put big /tmp/big

200 PORT command okay.

150 Opening data connection for $/ \mathrm{tmp} / \mathrm{blg}(146.137 .64 .1,2741)$.

226 Transfer complete.

local: blg remote: /tmp/big

1113494 bytes sent In 20 seconds (54 Kbytes/s)

ftp $>$ quit

221 Logged off. unstructured text flle transfer

The following is a sample of a Sun FTAM client to IBM FTP server session via the OSIMF/6000 FTAM/FTP gateway. First, the user initiates FTAM from the machine "olivia" (Sun), sets the concurrency variable off and the qualifier variable to ftpstore, opens the association with the machine "gateway" (IBM), transfers the file/tmp/big, and exits the FTAM program.

ollvla\%ftam

ftam > set concurrency off

concurrency = off - concurrency control

ftam $>$ set qualifier ftpstore

qualifler = "ftpstore" - service quallfier

ftam > open gateway

user (gateway:b32357): osldemo@hamlet

password (gateway:osidemo@hamiet):

gateway... connected

gateway> get $/ \mathrm{tmp} / \mathrm{big} / \mathrm{tmp} / \mathrm{big}$

ftam: 1093384 bytes recelved in 18.27 seconds (58.44 Kbytes/s)

gateway $=$ quit 
Table 9 lists the FTP commands valid over the FTP-to-FTAM side of the gateway.

TABLE 9 FTP Commands Accepted by the IBM FTAM/FTP Gateway

append--append a local file to a file on the remote machine

ascll--set representation type to network ASCII

binary--set representation type to image

close--terminate the session with the remote ftp server

delete--delete the file on the remote machine

get--retrieve the file and store it on the local machine

mdelete--delete the filles from the remote machine

mget-expand the files on the remote machine and do a get for each file

mput-expand the list of local files and do a put for each file

open--establish a connection to the specified host FTP server

put--store a local file on the remote machine

quil--terminate the session with the remote server and exit ftp

rename--rename the file on the remote machine

status--show the current status of the ftp session

\subsection{Interoperability}

We tested the functionality of the IBM FTAM/FTP gateway by initiating transfers between two Suns, between an IBM and a Sun, and between two IBMS. No difficulties were encountered performing these tests.

\subsection{Performance}

To measure the performance of the FTAM/FTP gateway, a sample file (1,093,384 byies) was transferred between two Suns over a local area network via the IBM OSIMF/6000 gateway and between an IBM and a Sun via the IBM gateway. Table 10 summarizes test results of the FTAM/FTP gateway. The performance of the gateway was slightly better than the performance of the pure FTAM $<->$ FTAM transfers. The biggest drawback of the FTAM/FTP gateway is the user interface. At Argonne, we feel there is a need for gateways of this type to avoid the need to install FTAM on all machines; however, our goal is to have a more transparent user interface. We will investigate improving the user interface of the FTAM/FTP gateway. 
TABLE 10 FTAM/FTP Gateway Transfer Times (Kilobytes/second)

Transfer Type

Sun FTAM->IBM gateway->Sun FTP(get)

Sun FTAM ->IBM gateway->Sun FTP(put)

Sun FTAM->IBM gateway->IBM FTP(get)

Sun FTAM->IBM gateway->IBM FTP(put)

Sun FTP->IBM gateway->Sun FTAM(get)

Sun FTP->IBM gateway->IBM FTAM(put)

Sun FTP->IBM gateway->Sun FTAM(put)

Sun FTP->IBM gateway->IBM FTAM(get)

IBM FTAM->IBM gateway->Sun FTP(get)

IBM FTAM->IBM gateway->Sun FTP(put)

IBM FTP->IBM gateway->Sun FTAM(get)

IBM FTP->IBM gateway->Sun FTAM(put)
File Type

binary

79.7

ascil

106.0

77.0

137.9

142.5

77.7

94.0

87.0

44.7

41.0

139.6

94.3
69.3

78.4

82.8

77.4

130.0

67.8

78.8

103.5

32.8

41.4

74.6

61.9 


\subsection{Future Testing}

Additional GOSIP testing remains to be completed at Argonne before we can complete the implementation of our GOSIP transition plan. Our next phase of investigation will involve testing the interoperablity of the Message Handling Systems $(X .400)$ provided by IBM OSIMF/6000 and SunNet MHS. Both products provide full-service Message Handling Systems, Message Transfer Agents, and $X .400 / S M T P$ gateway applications. We will also investigate electronic mail gateways from other vendors such as Retix. We will investigate the use of X.25 lower layer transport protocols, and the use of FTS2000 Message Handling Service by installing a gateway between a network that handles CLNP packets (e.g., the Internet) and one that handles CLNP packets encapsulated in X.25 (e.g., the FTS2000 Packet Switched Service). Other areas we plan to investigate in the future include Directory Services (X.500), Virtual Terminal Services, and Network Management products. This document will be revised as our testing progresses. 


\subsection{Appendix A. OSI Addressing}

An OSI address consists of a Network Service Access Point (NSAP) plus the selectors required to specify the destination entity within the system identified by the rest of the NSAP. An NSAP address is analogous to an Internet (IP) address. Selectors are similar to TCP port numbers in the TCP/IP sulte. ISO8348/Addendum 2 defines the semantics of the NSAP [2]. The principle concept is based on hierarchical addressing domains. An addressing domain is a set of addresses administered by a common authority that specifies the structure and ensures the uniqueness of identiflers within the domain. An addressing domain also shares common intra-domain routing protocols. The GOSIP Version 2 NSAP address format is shown in Table 11.

TABLE 11 GOSIP Version 2. NSAP Structure

\begin{tabular}{|c|c|c|c|c|c|c|c|c|c|}
\hline \multirow{3}{*}{$\begin{array}{r}\text { ISO NSAP } \\
\text { US Government IDI }\end{array}$} & \multicolumn{2}{|c|}{ IDP } & \multicolumn{5}{|c|}{ HO-DSP } & & \\
\hline & AFI & $|D|$ & \multicolumn{5}{|c|}{ DSP } & & \\
\hline & 47 & 0005 & DFI & $A A$ & Res & RD & Area & System ID & NSEL \\
\hline ! octets & 1 & 2 & 1 & 3 & 2 & 2 & 2 & 6 & 1 \\
\hline & & & $\begin{array}{l}\text { IDP-I } \\
\text { OO-DS } \\
\text { AFI - A } \\
\text { IDI - In } \\
\text { DSP. } \\
D F 1 \text { - } \\
\text { AA - A } \\
\text { Res - } \\
\text { RD - R } \\
\text { Area - } \\
\text { Systen } \\
\text { NSEL }\end{array}$ & $\begin{array}{l}1 \text { Do } \\
\text { - Hig } \\
\text { iority } \\
\text { i Don } \\
\text { main } \\
\text { man Fon } \\
\text { inist } \\
\text { erved } \\
\text { ting } \\
\text { ald } \\
\text { S S } \\
\text { SAP }\end{array}$ & $\begin{array}{l}\text { Part } \\
\text { der D } \\
\text { Form } \\
\text { Ident } \\
\text { cific } \\
\text { dent } \\
\text { Auth } \\
\text { Auturo } \\
\text { ain } \\
\text { er } \\
n \text { lder } \\
\text { ctor }\end{array}$ & Spe & 更 & & \\
\hline
\end{tabular}

The NSAP is made up of the initial domain part (IDP) and the domain specific part (DSP). The IDP identifles the authority responsible for the structure and assignment of the remainder of the address, the DSP. The IDP consists of an authority and format identifier (AFI) and an initial domain identifier (IDI). The AFI specifles the format of the IDI, the authority responsible for allocation of IDI values, and the syntax of the DSP.

The recommended DSP format for use in the U.S. portion of the Internet is under the IDP format administered by the General Services Administration (GSA) for the National Institute of Standards and Technology (NIST) [4] under Section 5.1.1 of GOSIP Version 2 [5]. iVIST has been designated as the authority to administer the addressing domain identifled by $|D|=0005$ under $A F \mid=47$. The AFI value 47 specifies that the IDI part is interpreted as a four decimal digit International Code Designator (ICD), and the DSP has a binary abstract syntax. The addressing domain under IDI=0005 is available for use by all of the U.S. federal government. NIST will specify the structure of the DSP under $|D|=0005$ and delegate the task of assigning DSP values to GSA. 
Within the DSP, the DSP Format Identifier (DFI) specifles the structure, semantics and administration requirements associated with the remainder of the DSP. Currently, only one DSP format $(D F \mid=80)$ is defined under $|D|=0005$.

The administrative authority identifles the entity responsible for the organization of routing domains, establishes policies that govern the information collected and disseminated internally and externally, esiabhlishes subdomains (areas), and delegates the corresponding responsibilities. A routing domain (RD) is a so! af intermediate systems (routers) and end systems (hosts) that operate according to the same routing procedures and are wholly contained within a single administrative domain. An administrative domain is a collection of end systems and intermediate systems administered by a single authority [6]. 


\subsection{Appendix B. The Argonne OSI Addressing Architecture}

The purpose of this appendli is to specify the Implementation of OSI address assignments, connecthonless network protocol (CLNP) service, and the associated routing protosols to be deployed by Argonne National Laboratory. This is a description of Argonne's proposed CLNP architecture, which is open to review and comments.

\subsection{Background}

Argonne is a contractor to the U.S. Department of Energy and therefore falls with the auspices of Government Open Systems Interconnect Profile (GOSIP) compllance. Argonne is currently connected to two major national backbones (NSFnet, ESnet) and to a mid-level regional network (CICNet) (see Figure 6). Many Argonne hosts also participate in HEPnet, a wide-area DECnet-based network. Argonne is located at two sites: Argonne, Illinols just outside Chicago and Argonne-West in Idaho Falls, Idaho. The IIIInois site is responsible for all Internet connectivity for both sites. The IIIInois and Idaho sites are interconnected by a private dedicated link. Backdoor connections at the Idaho site are strictly for the use of Argonne-West.

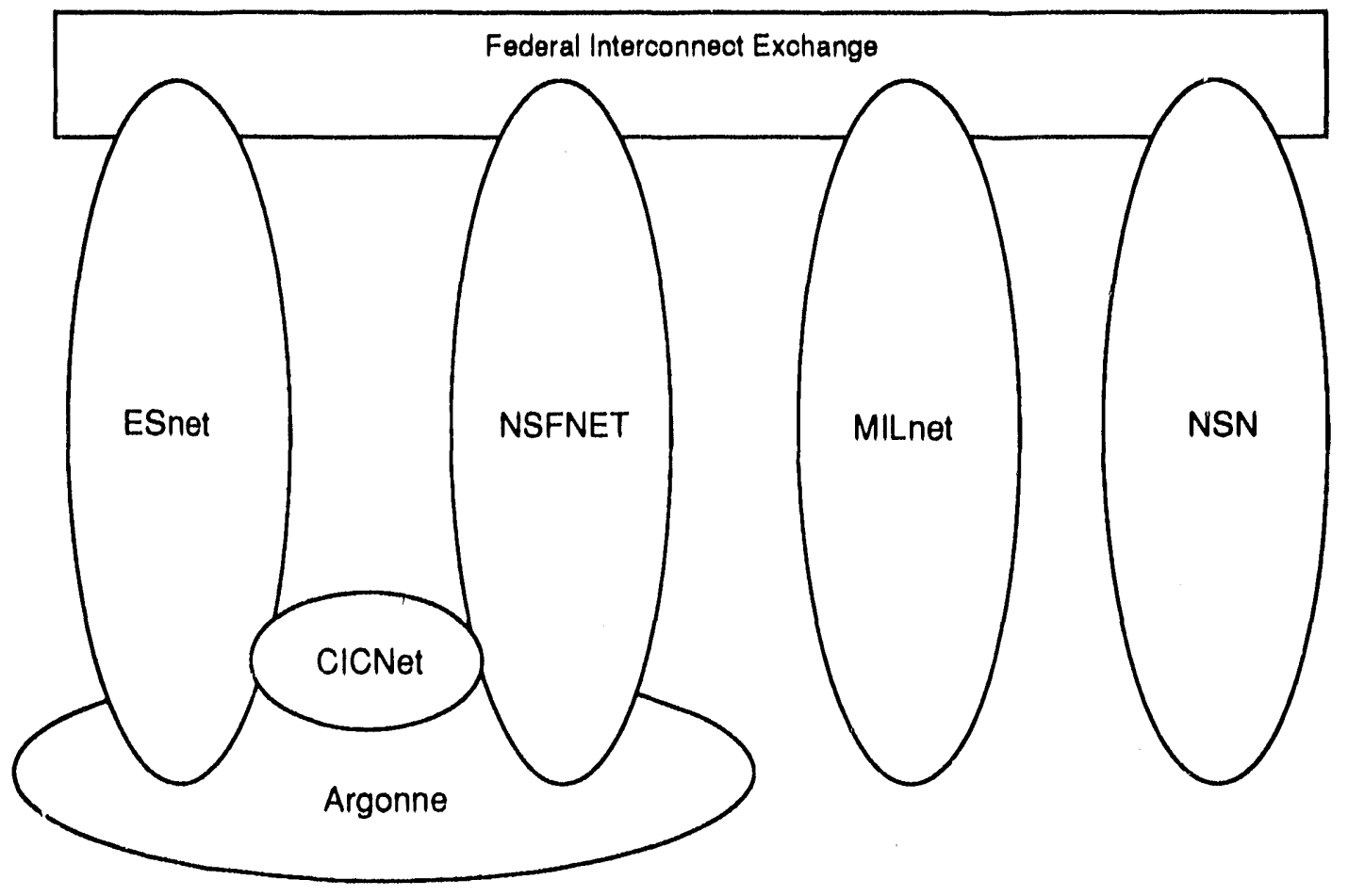

FIGURE 6

Argonne Internet Connectivity 


\subsection{Alternatives}

Argonne is faced with the following choices for NSAP address assignments within the U.S. Government Initial Domain Identifier (AFI=47 IDI=0005):

1. apply for its own Administrative Authority (AA).

2. apply for a Routing Domain Identifier (RD) within the CICNet AA (FFF400).

3. apply for a RD within the NSFnet AA (FFFF00).

4. apply for a RD within the ESnet AA (005700).

5. apply for a RD from the DOE AA (000400).

Alternative 1 represents the most costly approach to the Internet community as a whole. This approach would require that all transit routing domains carry a route to Argonne's AA (much like IP network numbers today). Alternatives 2 through 4 all bear the advantage of route aggregation. It is not clear who is administering routing in the case of Alternative 5.

\subsection{Argonne Strategy}

Argonne has elected to implement Alternative 4 because ESnet is our preferred ("primary") carrier. Given this decision, Argonne will negotiate with its other carriers (NSFnet and CICNet) to carry explicit NSAP prefix information defining Argonne's direct connection to each of these networks. Failure to do so would result in traffic to Argonne being routed via network interconnect points to ESnet rather than directly via the Argonne node on these backbones. For example, NSFnet traffic to Argonne would traverse NSFnet to one of the two Federal Interconnect Exchanges (FIX) where it would be handed off to ESnet for delivery to Argonne. CICNet traffic to Argonne would traverse CICNet to the CICNet/ESnet peering point at Argonne, where the ESnet router would send it to Argonne. Many of the major Internet players have informally agreed that carrying such explicit NSAP prefix information will be possible since the number of these exception cases is expected to be few. Future topologies may change this "gentleman's understanding" and call for further analysis.

Argonne will exchange routing information with ESnet, NSFnet and CICNet routers on a firewall boundary located at Argonne. The Argonne firewall router of choice is Cisco. At this time, Cisco has not implemented IS-IS or the Inter-Domain Routing Protocol. When these protocols become available, Argonne will investigate the use of these protocols. Until then, Argonne will use Cisco's ISO IGRP to exchange routing information with ESnet and CICNet (both use Cisco routers). Argonne's NSFnet connection is a T3 ENSS. CLNP service is not currently available on the T3 NSFnet backbone. In order for Argonne CLNP traffic to reach NSFnet sites, Argonne will use ESnet to reach a FIX where the packets will be directed to a T1 NSS. It is anticipated that the NSFnet T3 backbone will have CLNP service before the summer of 1992. It is desirable at that time to run IDRP with the NSFnet backbone.

Although the objective of this document is to deal with addressing and routing issues for CLNP, there are concerns about the transition from DECnet Phase IV to Phase V. Argonne currently utilizes Cisco routers which implement CLNP and DECnet Phase IV in parallel protocol stacks. This means tr.dt DECnet Phase IV and CLNP can coexist with completely separate routing tables. The problems begin when you try to ensure interoperability between DECnet Phase IV nodes and DECriet Phase $V$ nodes. Although the Digital Equipment Corporation and Cisco Systems, Inc. have each provided a 
solution to the Phase IV/Phase V interoperability problem, neither solution is suitable in the wide-area DECnet community. The wide-area DECnet routing scheme currently employed by HEPnet relies heavily on hidden addresses (nodes and areas) and encompasses geographically dispersed sites which share a DECnet Phase IV area. Experts within the ESnet and HEPnet communities are working on a solution. This document will be revised as solutions are identified.

\subsection{DECnet Phase V}

Argonne has a substantial investment in DIgital Equipment Corporation (DEC) systems. Until recently, these machines communicated using the proprietary DECnet protocol developed by DEC. DEC's announcement of ADVANTAGE Networks, Digital's fifth generation of networking products, implements and integrates OSI, TCP/IP, and DECnet networking protocols. With ADVANTAGE networks, Digital expands and opens the Digital environment by supporting the equipment of many vendors in a heterogeneous network.

DECnet /OSI Phase V provides many new features, including:

- backward compatibility with DECnet Phase IV systems

- expanded addressing capability

- OSI applications support to include FTAM and X.400 initially

- distributed name service to store node names and obtain protocol information

- ISO routing layer support

DECnet/OSI will eliminate the address limitations of DECnet Phase IV (1023 nodes per area and 63 areas per network) by incorporating OSI addressing. The DECnet/OSI Phase V addressing scheme offers the following benefits:

- increased addressing capabilities

- backward compatibility with Phase IV addressing

- conformance to ISO 8348/AD2 addressing standard

Argonne has a large DECnet Phase IV network that is coordinated with the High Energy Physics wide-area DECnet network (HEPnet). To maintain interoperability and connectivity to HEPnet during a transition from Phase IV to Phase V, extensive planning is under way. Essentially, DECnet networks will have to transition an area at a time. In a transition, for a Phase $V$ node to communicate with a Phase IV node, the Phase V node must maintain a Phase IV-compatible address. In a transition, it will be necessary to assign Phase $V$ nodes multiple addresses (called multihoming). One address will be a Phase IV compatible address, and one will be a true Phase V address. Once the network has successfully made the transition to Phase $V$, the Phase IV compatible address may be dropped.

DECnet/OSI for ULTRIX is available. DECnet/OSI for VMS, however, is in the beta test phase at this time. Argonne has a large investment in the VMS/DECnet environment. We hope to be able to work with DECnet Phase V products for VMS before the end of FY1992. 


\subsection{Internet OSI Activity}

The Internet is the collection of approximately 5,000 independent packet switched networks interconnected by routers. Today, these 5,000 networks are made up of private campus and business LAN complexes, public data networks, government and government-sponsored networks (U.S. and others). During its initial development, the Internet consisted of a set of research and education networks that switched Internet Protocol (IP) packets on a global basis. More recently, multi-protocol router technology has emerged which permits parts of the Internet to support not only the TCP/IP protocol suite, but the OSI Connectionless Network Protocol and a variety of proprietary protocols (e.g., Novell IPX, AppleTalk, DECnet, etc.). The multiprotocol capability of the Internet is not uniformly deployed, however, and the most extensive facilities are still based on the IP protocol. It is likely that more network service providers will offer OSI services as the demand for such services increases. To date, the demand has been slow in developing for OSI services.

Significant portions of the Internet are now operated without limitation as to the kinds of traffic sent (content, purpose). The commercial IP service providers in the U.S. and Europe (e.g., Performance Systems international, Advanced Network and Services, UUNET/Alternet, Infonet, etc.) allow any uses. The U.S. government-sponsored portions of the Internet (NSFnet, NSInet, ESnet and some of the U.S. intermediate-level networks such as CICNet, and SURAnet) have various constraints on the traffic they are willing to carry. In the last two years, there has been a relaxation of some restrictions, for instance in the NSFnet backbone, to allow commercial service providers (electronic mail, on-line information) to offer service to the users of the NSFnet backbone (notably, the intermediate-level networks) as long as the end users of the service apply it to scholarly, research, and educational activity.

So, the Internet is a grand collaboration, growing by every metric (hosts, users, networks, traffic). There are over 700,000 hosts on the network (ranging from supercomputers to desktop/notebook computers). There are an uncertain number of users, but the number of Internet users has been estimated at over 4 million. Traffic is growing at a rate of 10-15\% per month (as measured in the NSFnet backbone by the operator, Merit Network, Inc.). Merit statistics for the month of January 1992 indicate over one billion packets accepted by the NSFnet backbone during the month. 


\subsection{Appendix C. Sample Isoentities File}

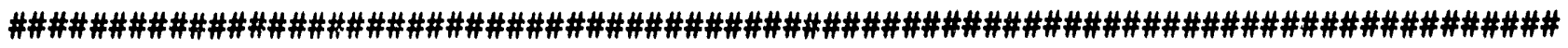
ISOENTITIES

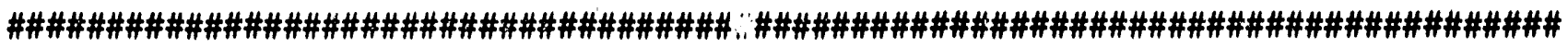

\# This filie constitutes the application entities title database that is used by the stub-directory service \# of the FTAM client application. In the absence of X.500, its purpose is to provide a mapping \# between the application entities on the OSI network and their presentation addresses. Each

\# application entity is identified by a pair of values, an object descriptor and an object identifier.

\# The object descriptor appears as a designator-qualifier value. The designator is a host name and \# the qualifier identifies a service provided by that host. The entries within this file are partitioned \# into two categories:

\# - templates for services

$\#$ - local services

\#

\# For system administrators, the only need to modify this file will result from the introduction of a \# new node onto the network. Each of these nodes should be placed within the latter category \# (local services). An example and description of this appears later within this file.

\# The syntax of each entry within this file is as follows:

\# <hostname> <service> <application_entity_title><presentation_address>

\# Each token is separated by white space (blanks and/or tabs), though double-quotes may be \# used to prevent separation. 
Appendix C. Sample Isoentities File

\#\#\#\#\#\#\#\#\#\#\#\#\#\#\#\#\#\#\#\#\#\#\#\#\#\#\#\#\#\#\#\#\#\#\#\#\#\#\#\#\#\#\#\#\#\#\#\#\#\#\#\#\#\#\#\#\#\#\#\#\#\#\#\#\#\#\#\#\#\#\#\#\#\#\#\#\#\#\# \# TEMPLATES FOR SERVICES \#

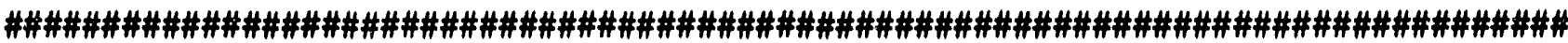

default ftpstore 1.3.9999.1.7 \#523/

default filestore 1.3.9999.1.7 \#259/

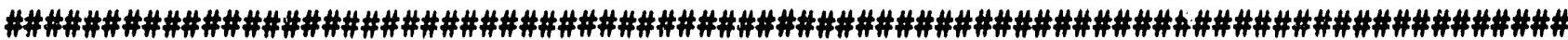

\# LOCAL SERVICES \#

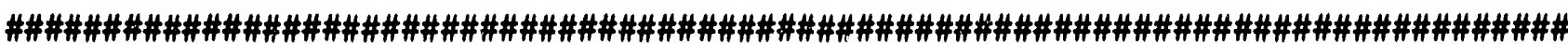

\# This section contains entries for each host on the network. A description of the fields within

\# each entry is given below:

\# Hostname The host name identifies a remote host on the OSI network. Its purpose is to allow \# an FTAM client user the ability to reference the remote host in a symbolic way. In other words, \# the existence of this permits the user to reference the host by name rather than by a \# complicated Presentation Address.

\#

\# Service This field identifies the service that is to be provided by the new remote host.

\#There are three possible values:

\# 1. filestore - denotes that the remote host will be acting as an FTAM server.

\# 2. ftpstore - denotes that the remote host

\# 3. default - denotes that the role of the remote host will be determined by the current value of \# the FTAM variable "qualifier".

\# Application this value must be obtained from the system adminstrator of that particular system. \# Note that a null AET may be specified as NULL. 
\# Presentation Presentation Addresses are formed by four values:

\# Address a presentation selector (PSEL), a session selector (SSEL), a transport selector (TSEL), \# and a network address. The network address of each host must appear in the \# form NS+<network_address >.

$\#$

\# The Argonne ESs are shown below.

hamlet default 1.3.9999.1.7 NS+4700058000570000000010c00002608c2fe9f501

gateway default 1.3.9999.1.7 NS+4700058000570000000010c00002608c2fe9f501

olivia default NULL NS+4700058000570000000010c00008002009057600

olivia mta NULL "MHS"/NS+4700058000570000000010c00008002009057600

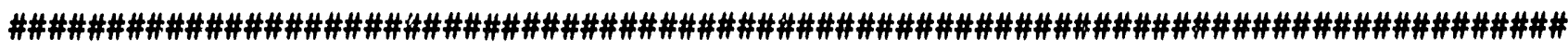

\# A Sun located at Lawrence Livermore National Lab (ESNET)

marmot default NULL NS+47000580005700000001E00n01aa0004002cd200 


\subsection{References}

[1] Information Processing Systems- Data Communications- Network Service Definition. International Organization for Standardization and International Electrotechnical Committee, April, 1987. International Standard 8348.

[2] Information Processing Systems- Data Communications - Network Service Definition Addendum 2: Network Layer Addressing. International Organization for Standardization and International Electrotechnical Committee, March, 1988. International Standard 8348/Addendum 2.

[3] Information Technology - Telecommunications and Information exchange between systems - OSI Routeing Framework, International Organization for Standardization and International Electrotechnical Committee, July, 1989. TR 9575.

[4] OSI NSAP Address Format For Use In The Internet. P. Colella, R. Callon. Internet-Draft, Internet Engineering Task Force Network Working Group, February 12, 1991.

[5] GOSIP Advanced Requirements Group. Government Open Systems Interconnect Profile (GOSIP) Version 2. Federal Information Processing Standard, U.S. Department of Commerce, National Institute of Standards and Technology, Gaithersburg, MD.

[6] Information Processing Systems- Telecommunications and Information Exchange Between Systems- End System to Intermediate System Routing Exchange Protocol for Use in Conjunction with the Protocol for Providing the Connectionless-mode Network Service. International Organization for Standardization and International Electrotechnical Committee, August, 1988. International Standard 9542.

[7] Information Processing Systerns- Telecommunications and Information Exchange Between Systems-Intermediate System to Intermediate System Intra-Domain routeing exchange protocol for use in Conjunction with the Protocol for providing the Connectionless-mode Network Service. International Organization for Standardization and International Electrotechnical Committee, February, 1990. Draft Proposal 10589.

[8] Information Processing Systems- Teiecommunications and Information Exchange Between Systems- Intermediate System to Intermediate System Inter-Domain Routeing Exchange Protocol. International Organization for Standardization and International Electrotechnical Committee, July, 1991. Draft International Standard 10747.

[9] Information Processing Systems- Data Communications- Protocol for Providing the Connectionless-Mode Network Service. International Organization for Standardization and International Electrotechnical Committee, December, 1988. International Standard 8473.

[10] AIX Version 3 for RISC System/6000 OSI Messaging and Filling/6000 User's anci System Administrator's Guide. IBM, September, 1991. SC32-0012-01. 
[11] SunNet OSI Installation Gulde. Sun Microsystems, February, 1991. Sun Microsystems, Inc. 800-5080-10.

[12] SunNet OSI System Administration Manual. Sun Microsystems, February, 1991. Sun Miorosystems, Inc. 800-5006-10.

[13] SunNet OSI FTAM User's Gulde. Sun Microsystems, February, 1991. Sun Microsystems, Inc. 800-5803-10.

[14] Information Processing Systems- Open Systems Interconnection- File Transfer, Access and Management. International Organization for Standardization and International Electrotechnical Com. mittee, October, 1988. International Standard 8571.

[15] Request for Comment 1139 An Echo Function for ISO 8473. R. Hagens, January, 1990. 

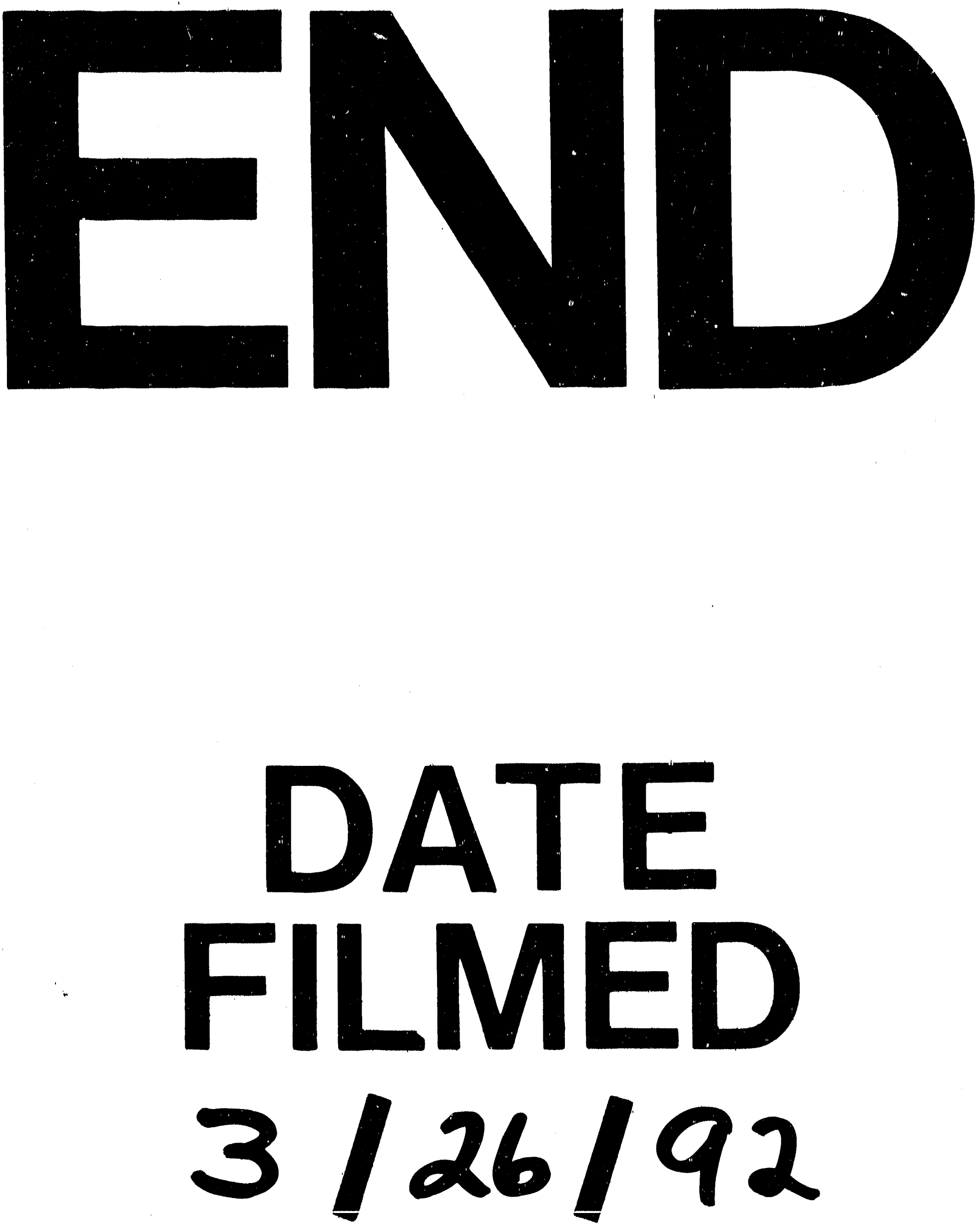
